\title{
FLICKER DISTORTS VISUAL SPACE CONSTANCY
}

\author{
Stephen L. Macknik, ${ }^{*}$ Brian D. Fisher $\nmid$ and Bruce Bridgeman $\ddagger$ \\ Psychology Board of Studies, Kerr Hall UCSC, Santa Cruz, CA 95064, U.S.A.
}

(Received 3 October 1990; in revised form 24 April 1991)

\begin{abstract}
Effects of flicker on space perception were measured by displacing a flickering target during saccadic eye movements. A small target was flickered at 33, 66, 130 or $260 \mathrm{~Hz}$. Using a 2-interval forced-choice design, sensitivity to the displacement was about twice as great when the target was moved in the direction opposite the eye movement as when it was moved in the same direction. This would be expected from a partial breakdown of space constancy - the world should seem to jump in the direction opposite an eye movement. Even if a suppression of displacement detection during saccades prevents this jump from being perceived, it should be easier to detect a target displacement in the direction opposite the eye movement than in the same direction: when movement is opposite, the imposed displacement adds to the illusory displacement, making detection easier. Displacements were more easily detected at lower flicker rates. Results imply that both masking and extraretinal signals are important in suppressing the detectability of target displacements during saccades, and that flicker on video display terminals may distort space perception.
\end{abstract}

Flicker Space constancy Saccadic suppression VDT Saccades Efference copy

Space constancy, the perception that the world has remained stable when the eyes move, depends on extraretinal signals. One of the extraretinal signal theories, the classical idea of efference copy, assumed that a compensatory outflow signal exactly equalled the change in retinal signal during an eye movement (von Holst \& Mittelstaedt, 1950; Sperry, 1950). Teuber (1960) named the efference copy theories "cancellation theories" because they imply a feedforward cancellation of the retinal motion signal. There is a flaw built into these theories; it is the requirement that the extraretinal signal must provide perfect compensation for the retinal image displacement during an eye movement. The signal must support the introspection that the world does not seem to move in the slightest when the eye jumps. But all biological signals are noisy-they wander with time, they depend to some extent on the current state of arousal, health, and nutrition, and they will vary slightly from one movement to the next. Thus, though such signals clearly play a role

*Present address: Program in Neurosciences, Harvard Medical School, 25 Shattuck Avenue, Cambridge, MA 02115, U.S.A.

†Present address: Department of Psychology, Queen's University, London, Ontario, Canada.

\$To whom reprint requests should be addressed. in constancy, they cannot support constancy unaided.

Efference copy has, in fact, been shown to have a gain of $<1$ (Yasui \& Young, 1975a, b; Grüsser, Krizic \& Weiss, 1987; Pola \& Wyatt, 1989). But efference copy is not the only source of information about eye position, for a small proprioceptive component may be present as well (Gauthier, Nommay \& Vercher, 1990). Efference copy and proprioception sum to create a composite extraretinal signal (Matin, 1972). But recent experiments that measure proprioception and efference copy separately in the same subjects show that during saccades even this sum has a gain of $<1$ (Bridgeman \& Stark, 1991).

This finding suggests that extraretinal signals must be supplemented by other mechanisms to achieve space constancy and to stabilize perceived visual direction. A mechanism that raised the threshold for perceiving displacement of the world during a saccade could mask the error in the extraretinal signal and allow the impression of a stable world despite incomplete extraretinal signal compensation.

Many laboratories have described such an increase in threshold for detecting image displacements during saccadic eye movements. Saccadic suppression was first described by Dodge (1900) as a suppression of visibility of a 
flashed target during a saccade, and early work on the phenomenon was limited to flash suppression. The magnitude of the flash effect is $<0.7$ log units (Latour, 1962; Volkmann, Riggs, White \& Moore, 1978), and even that is obtained only near threshold. Saccadic suppression of displacement detection, in contrast, reaches at least $4 \log$ units (Bridgeman, Hendry \& Stark, 1975; Mack, 1970; Stark, Kong, Schwartz, Hendry \& Bridgeman, 1976), and is obtained even for displacements of several degrees. Saccadic suppression of displacement makes up for errors in extraretinal signals, suppressing retinal motion signals that otherwise would cause perception of displacement of the visual field with each saccadic eye movement. This paper addresses the question: how sensi-tive is saccadic suppression of displacement to the dynamic structure of the image during a saccade?

Bridgeman et al. (1975) suggested that saccadic suppression of image displacement (SSID) aids the extraretinal signal in achieving space constancy. They did the first parametric study of the phenomenon, varying both target displacement and size of eye movement, and using an event detection paradigm. The degree of suppression depended upon the ratio of eye movement to target displacement, and not upon the absolute size of either. No directional asymmetry was found; displacements in the same direction as the saccade yielded identical thresholds to displacements in the opposite direction. A follow-up study extended this result to orthogonal as well as collinear directions of motion, and found again that the dircetion of motion did not affect thresholds (Stark et al., 1976).

During a $10^{\circ}$ saccade, the eye moves at a peak velocity of about $500^{\circ} / \mathrm{sec}$. At this speed a clear image cannot be transduced; instead, a smeared image is painted on the retina. In continuous lighting this smeared image is not perceived; an inhibitory process of backward masking, similar to metacontrast, prevents perception by using the image available after fixation is reestablished to mask the smear (Grüsser, 1972; Matin, Clymer \& Matin, 1972).

Two types of signal might affect SSID: extraretinal signals such as efference copy and proprioception, and retinal signals such as smear of an image during a saccade. Some authors have concluded that only smear contributes to saccadic suppression (Brooks \& Fuchs, 1975). while others assign a dominant role to extra retinal signals (Li \& Matin, 1990). The role of smear was studied by Festinger and Holtzman (1978), who asked subjects to estimate the amount of displacement of a target during a saccade. The display was on an oscilloscope refreshed at $1 \mathrm{kHz}$. When smear was eliminated by blanking the display during a saccade or by moving the display along with the eye, subjects detected a smaller proportion of the image displacement, as though they perceived their own eye movements to be smaller than they really were. Uncertainty, measured as SD, also increased when smear was eliminated. These results suggest that smear plays a role in SSID; since no smear occurred on the retina, only the extraretinal signal could mediate saccadic suppression. And because the gain of the extraretinal signal is $<1$, the compensation of the image was less than the veridically required amount.

Some controversy remains, however, about how much effect smear has on SSID. Li and Matin (1990) suggest that smear plays no part in the last $75 \%$ of a $10^{\circ}$ saccade. Although this seems to contradict the findings of Festinger and Holtzman (1978), an alternative explanation may exist. Simply put, $\mathrm{Li}$ and Matin did not measure smear information from the first $25 \%$ of the duration of their saccades. While they showed persuasively that postsaccadic exposure duration affects displacement threshold, and that smear information from the final $75 \%$ of a $10^{\circ}$ saccade is not significant, $\mathrm{Li}$ and Matin did not show that the role of smear is insignificant throughout the saccade.

All of the experiments reviewed to this point were performed under continuous lighting conditions, or with sampling so fast as to be continuous for physiological purposes. By vary. ing the temporal sampling and spatial sampling of a stimulus, flickering displays may be able to disambiguate some of the issues that are still unresolved in defining the mechanisms of SSID. Smear can be varied by changing the flicker frequency while oculomotor behavior and the appearance of the image during fixation remain the same. Flicker implies that at a given instant in time, perception relies not on presently available information but rather on visual persistence from the previously flashed sample. When the eye is moving relative to the image, this temporal delay results in reduction of smear and in spatial distortion.

A flickering target remains spatially undistorted as long as the eye does not move, so that its location is well defined. During a saccade, however, the available information about stimu- 
lus location will lag behind the eye position. Since the information from a flickering stimulus is not available during the inter-flash interval, visual perception must depend upon persistence of the most recent flash. Just before the next sample of a flickering target, for example, only information from the previous flash is available. Also, the stimulus does not present a continuous streak across the retina during a saccade, but rather a series of spatially discrete samples. Both the spatial distortion and the temporal discontinuity can be varied quantitatively by varying the flicker rate and the duty cycle. In our analysis flicker can still affect perception during eye movements even when it is above the critical flicker fusion frequency, because it changes spatial sampling on the retina.

\section{METHODS}

\section{Subjects}

The 6 subjects were 3 undergraduates, naive about the hypothesis of the experiment, and 3 experienced psychophysical observers. Naive subjects were paid students at the University of California, Santa Cruz. All subjects were informed that minor eye fatigue might result from the experiments.

\section{Apparatus}

The monocular target stimulus was presented on the screen of a HP 1351 vector display with a medium-short persistence $\mathrm{p} 31$ phosphor. This phosphor decays exponentially to $1 \%$ brightness in $0.02-2$ msec (Keller, 1983). Since we ran our experiment at a brightness of $2 \mathrm{log}$ units over threshold, the stimulus always decayed to invisibility in $2 \mathrm{msec}$ or less. This brightness level also prevents any long-persistence phosphorescence of the screen from affecting perception.

The screen was placed $58.5 \mathrm{~cm}$ away from the subject's eye. It was $43.5^{\circ}$ wide and $32.2^{\circ}$ in height, and was of uniform brightness. Ambient light level at the observer's eye was $0.12 \mathrm{~cd} / \mathrm{m}^{2}$ ( 5 measurements, $\mathrm{SD}=0.03$ ). The display was controlled and timed by a PDP $11 / 23$ computer via a HP 1351 vector graphics display buffer. Duty cycle was held constant at $50 \%$ over all flicker frequencies. Subjective brightness was the same for all conditions. A Wavetek digital function generator served to trigger the sampled input. Subjects responded on a button box assembly, signalling the computer.
Eye movements were recorded with a photoelectric infrared eye-tracking system and sampled by the computer. An infrared LED illuminated the viewing eye, and paired photocells were aimed at the iris-sclera border. Outputs of the photocells were differentially amplified to give a dynamically accurate record of horizontal eye position. The subject's head was restrained with a bite bar assembly to which the eye tracking photocells and LED were attached. Experimenters monitored all eye movements during trials with a second oscilloscope.

\section{Procedure}

Using a two-interval forced choice paradigm, we tested displacement discrimination in either direction during a saccade in a flickering environment. The target was displaced without motion; once a saccade was detected, the target was extinguished and redrawn at a different position. The stimuli varied in flicker frequency, displacement distance, direction of displacement, and position on the screen for each trial.

At the start of a trial, the subject saw 3 outline squares on the screen, $20^{\circ}$ separating the left and right squares. The center "target" square was located midway between the flanking "fixation" squares (Fig. 1, panel A). The subject fixated
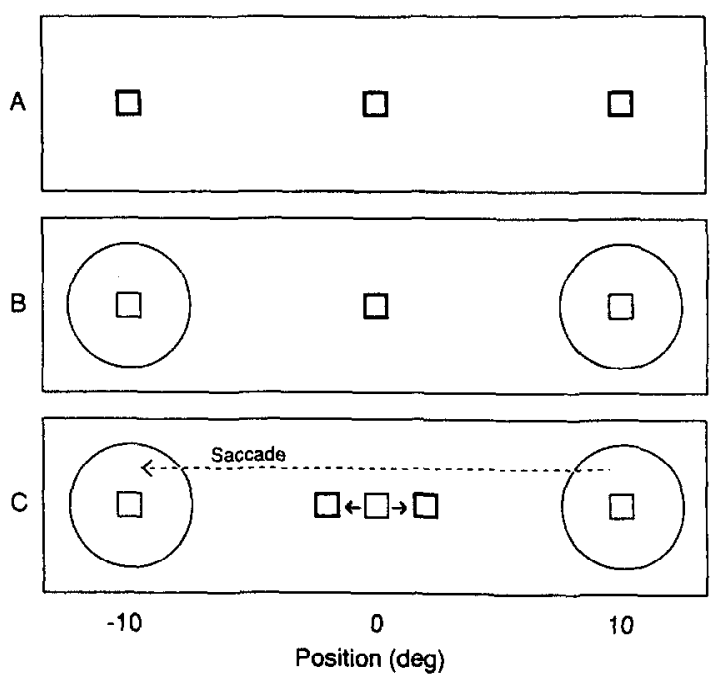

Fig. 1. Stimulus conditions during a trial. Heavy lines represent contours visible to the subject. At the start of a trial, 3 outline squares appear on the screen [panel $A$ ]. When the subject, fixates on the right square, it and the goal square on the left are extinguished [panel B]. During the saccade from the location of the right square to the location of the left square, only the center target is visible. The target can be displaced left or right by a variable amount [panel C]. 
the rightmost square and pressed a "ready" button. After a variable period, the left and right squares disappeared. This was the signal to saccade to the previous location of the left square (Fig. 1, panel B). Three seconds after the saccade, the fixation squares reappeared for a second interval, the subject again fixated the rightmost square, and the saccade was repeated. During either the first or the second interval, the center target square was displaced either leftward or rightward by one of 3 displacement sizes (Fig. 1, panel C). Displacement took place after about one-third of the saccade duration, near the peak of the saccadic suppression of displacement function (Bridgeman et al., 1975). The subject indicated with the button box whether the displacement occurred during the first or sccond interval of the pair. At the time of response, neither the target nor the fixation points were on the screen; these stimuli reappeared with the start of the next trial.

The subject's task was to report in which interval displacement occurred. The 576-trial experiment comprised 4 flicker frequencies $(33,66,130$ and $260 \mathrm{~Hz}) \times 3$ displacement sizes $\left(2.67,1.33\right.$ and $\left.0.67^{\circ}\right) \times 2$ directions of target displacement (left and right) $\times 12$ stimulus positions $\times 2$ trials of each type. Stimulus position was varied to eliminate the (admittedly unlikely) possibility that some sort of egocentric position cue could inform subjects whether the target had moved by monitoring the post-displacement position of the target. To change stimulus position, the target and both fixation points were offset by the same amount so that the geometry of the stimuli remained the same. Data were collapsed across these 12 stimulus positions so that each data point was based on 24 trials in each subject.

Trial order was randomized over all parameters. Each trial run was divided into 24 blocks of 24 trials, with a compulsory rest period between blocks. Each subject ran one practice block before data collection began. Figure 1, panel $\mathrm{C}$ is a general outline of an "action interval" (an interval in which target movement takes place). Target luminance was set at $2 \log$ units above threshold for each subject by viewing the target through a $99 \%$ neutral density filter and adjusting the luminance to threshold. Experimental runs were then performed without the filter. Target luminance was adjusted to appear equal at each flicker frequency.

\section{Eye movement monitoring}

Saccades were detected on-line as movements that exceeded $75^{\circ} / \mathrm{sec}$ for $16 \mathrm{msec}$. Every msec, this interval was probed by comparing the current digitized eye position sample with the sample collected $16 \mathrm{msec}$ earlier. Target offset occurred $100 \mathrm{msec}$ after saccade detection.

The eye movement monitor was calibrated before each session by presenting each of the 12 pre-saccade and 12 post-saccade fixation points in order. The points were taken from the 12 stimulus positions. The subject fixated each point in turn, pressing a button when fixation was achieved. The computer picked up 100 eye position samples $2 \mathrm{msec}$ apart, calculated a mean position and a SD to estimate fixation instability, and illuminated the next point. Unstable fixation resulted in an automatic restart of the calibration procedure. During the experimental sequence, piecewise linear interpolation was used to determine eye position between the calibration points.

Saccadic errors were detected by the computer and defective trials were redone. If the saccade was too short, too long, a double saccade occurred, or no saccade occurred, the subject saw an error message on the screen describing one of these 4 error conditions, and the trial was repeated later. If fixation remained outside the region of the initial fixation target, the subject received a "calibration problem" message on the screen. With some practice, most subjects could progress through the trials with few error messages.

\section{RESULTS}

Data are based on 3456 trials in 6 subjects. Figure 2 displays all results averaged over subjects. As target displacement increases, detectability increases (see also Fig. 3). Also, generally, displacements were easier to detect at low flicker frequencies than at high flicker frequencies (see also Figs 3 and 4). This occurred despite the fact that during fixation all ficker conditions appeared similar, except that some subjects were aware of more flicker at the slowest rate $(30 \mathrm{~Hz})$. Subjects generally did not know which flicker rate was being presented or even that flicker rate was an independent variable. This differs from normal experience, i.e. observing the flicker of a pilot lamp in darkness: in this high-contrast situation, the observer perceives the discrete samples in the retinal 


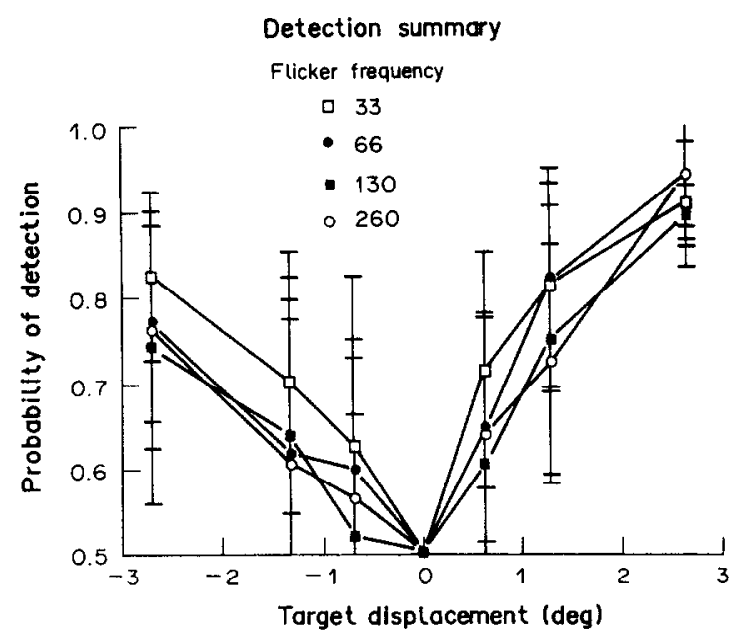

Fig. 2. Probability of detection vs target displacement for the 4 flicker frequencies. Negative target displacements denote target deviations to the left, in the same direction as the saccade. These wcrc more difficult to detect at all displacement amplitudes. Positive displacements denote target movement in the direction opposite the saccade.

after-image. Finally, target displacements in the opposite direction from the saccade (displayed as positive numbers on the $x$-axis in Fig. 2) were more easily detectable than target displacements in the same direction (see also Fig. 4).

In agreement with the saccadic suppression research done under continuous lighting conditions, analysis of variance (ANOVA) found a strong positive relation $\left(F_{[2,10]}=56.75\right.$, $P<0.001)$ between probability of detection and displacement size (Fig. 3). ANOVA also found flicker frequency to be negatively related to displacement detection $\left(F_{[3,15]}=5.636\right.$, $P<0.009$ ) (Fig. 3). A post-hoc test (Duncan, alpha $=0.05$ ) was performed on flicker frequency data (collapsed across displacement) to determine differences between flicker frequen-

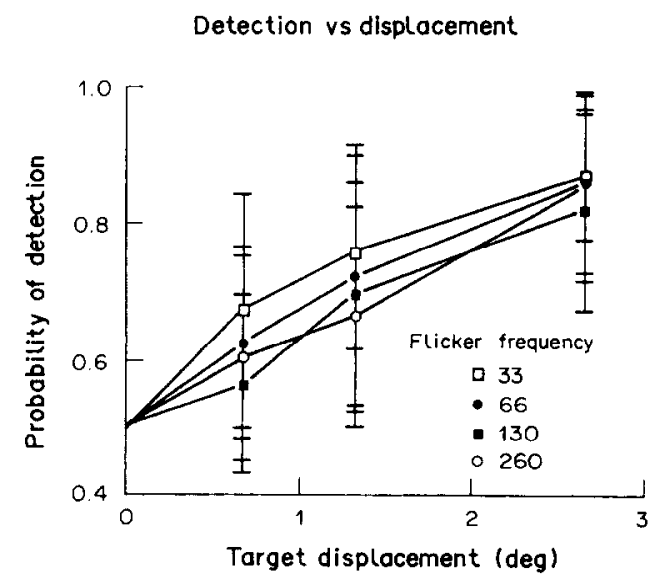

Fig. 3. Probability of detection vs target displacement for each flicker frequency, averaged over the two directions of target displacement.
Detection vs flicker frequency

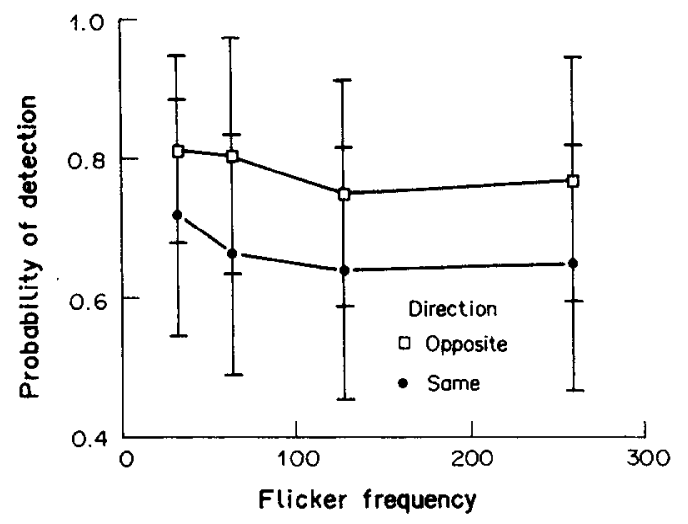

Fig. 4. Probability of detection for each of the 4 flicker frequencies, collapsed over displacement magnitude and direction.

cies. $130 \mathrm{~Hz}$ was shown to be insignificantly different from $260 \mathrm{~Hz}$, signifying that a ceiling effect begins to occur at a flicker frequency less than $130 \mathrm{~Hz}$, but greater than $66 \mathrm{~Hz}$. The Duncan test also confirmed that $33 \mathrm{~Hz}$ is significantly easier to detect than 130 and $260 \mathrm{~Hz}$.

Further, the analysis found that target displacements in the same direction as the eye are more easily detected than target displacements in the opposite direction $\left(F_{[1,5]}=10.635\right.$, $P<0.022$ ) (Fig. 4).

\section{DISCUSSION}

Figure 5 illustrates how retinal smear may relate to saccadic suppression of displacement.

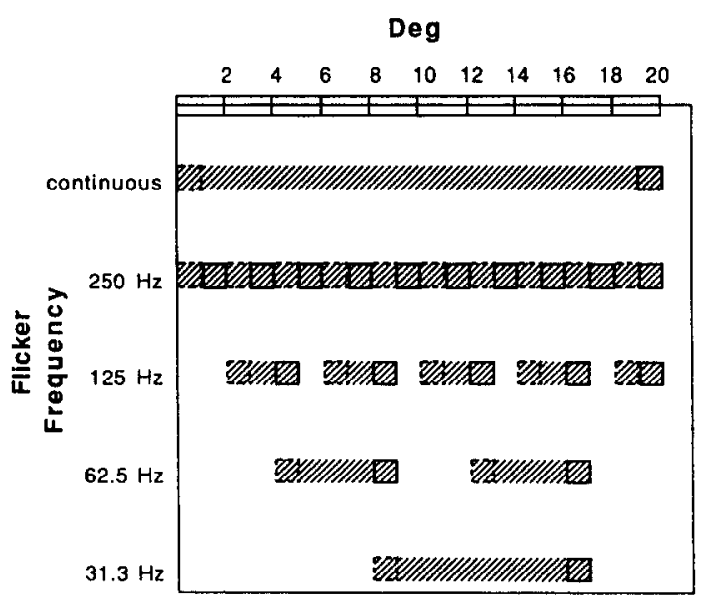

Fig. 5. Schematic representation of retinal images of a square stimulus, fixed on the screen, during a saccade of $500^{\circ} / \mathrm{sec}$. Each dashed square represents the position of a target at the beginning of a duty cycle, and each solid square represents the position at the end of the duty cycle. The diagonal hatching represents the smear on the retina when the target is on. When the stimulus is flickering at $62.5 \mathrm{~Hz}$., it appears once every $16 \mathrm{msec}$, and successive samples are $8^{\circ}$ apart on the retina. 
Under continuous lighting, a square stimulus $1^{\circ}$ on a side would smear continuously during a $20 \mathrm{deg}$ saccade. If the square were flickering at a rate of $250 \mathrm{~Hz}$, with an onset period (duty cycle) of $2 \mathrm{msec}$, a new smear element would be drawn every $4 \mathrm{msec}$ or every $2^{\circ}$ (assuming that peak saccade speed is about $500^{\circ} / \mathrm{sec}$ ). As flicker rate decreases, smear becomes more discontinuous as wider unstimulated areas appear, and samples are more separated spatially; if contiguity of smear is important in mediating saccadic suppression, we may expect saccadic suppression of displacement to decrease as well.

This analysis predicts that the lack of contiguity in smear from flickering displays should result in information that eye movements are smaller than they really are, and that targets should therefore seem to move in the direction opposite an eye movement. Thresholds for detecting target movement in the direction opposite the eye movements should decrease and false alarms should increase. Thus reduction of displacement detectability during saccades should be directionally biased and distortions of space constancy should be direction-specific. The spatial mislocalization of the target due to visual persistence of a previous sample would also tend to decrease detection thresholds, acting as though a real, if subthreshold, movement had occurred during the saccade. Even differences in flicker that are not perceived might influence the detectability of target displacements.

One might incorrectly assume that saccadic suppression of displacement is due entirely to spatial masking. Initially, spatial masking may appear to account for saccadic suppression: both phenomena are affected by retinal smear. However, Bridgeman (1983) showed that suppression is a different process from spatial masking by comparing retinal smear during a saccade to a similar retinal smear produced during fixation. The saccadic results were taken from a SSID study by Bridgeman and Stark (1979). In this experiment subjects made two successive $18^{\circ}$ saccades, during one of which a visual target was displaced $2^{\circ}$. The subjects's task was to detect which of the two saccades was accompanied by a target displacement. The smear on the retina from the saccade without target displacement was $18^{\circ}$, while smear from the other saccade was either 16 or $20^{\circ}$ depending on whether displacement occurred in the same or opposite direction as the eye movement. As expected, the $2^{\circ}$ displacement was undetectable. Bridgeman (1983) then tested the null hypothesis that detection thresholds during saccades and during fixation are identical. Bridgeman and Stark's (1979) conditions were simulated during fixation so that a similar retinal smear was produced without saccades. The results were clear: during fixation, subjects could detect differences in displacements as small as $15 \mathrm{~min}$ arc. Because near-identical retinal smear produced two drastically different effects during fixation and saccades, an internally driven saccadic suppression must exist in addition to spatial masking. Therefore, saccadic suppression of displacement is not accounted for by masking alone and the two systems are functionally distinct.

The current results imply that information concerning retinal sampling affects saccadic suppression of displacement. By flickering the display at different frequencies, we have decreased sampling by different amounts; at low flicker frequencies saccadic suppression of target displacement is incomplete, while at high flicker frequencies target displacement suppression is maintained (Fig. 3).

Another result of this study is that the suppression is asymmetric with respect to target displacement direction. Figure 4 is a representation of the probability of displacement detection vs flicker frequency for each direction of target movement. The difference between these two lines demonstrates a significantly higher detectability for target displacement opposite to the direction to the direction of the saccade than for the displacement in the same direction as the saccade. Figure 6 illustrates the basis of this asymmetry at the retina by presenting a sequence of trials flickering at varied rates. The top panel shows displacement in the same direction as the saccade, while the bottom panel shows displacement in the opposite direction. both overlaid with simulated retinal smear (saccades are performed from the left to the right fixation points). Displacement in the opposite direction decreases retinal sampling, and displacement in the same direction increases sampling. This is consistent with the theory that retinal smear is related to the differences we found in displacement detection thresholds; less smear resulted in lower thresholds for displacements during saccades.

The spatial sampling bias introduced by flicker during eyc movements also is consistent with a lower detection threshold when the target 


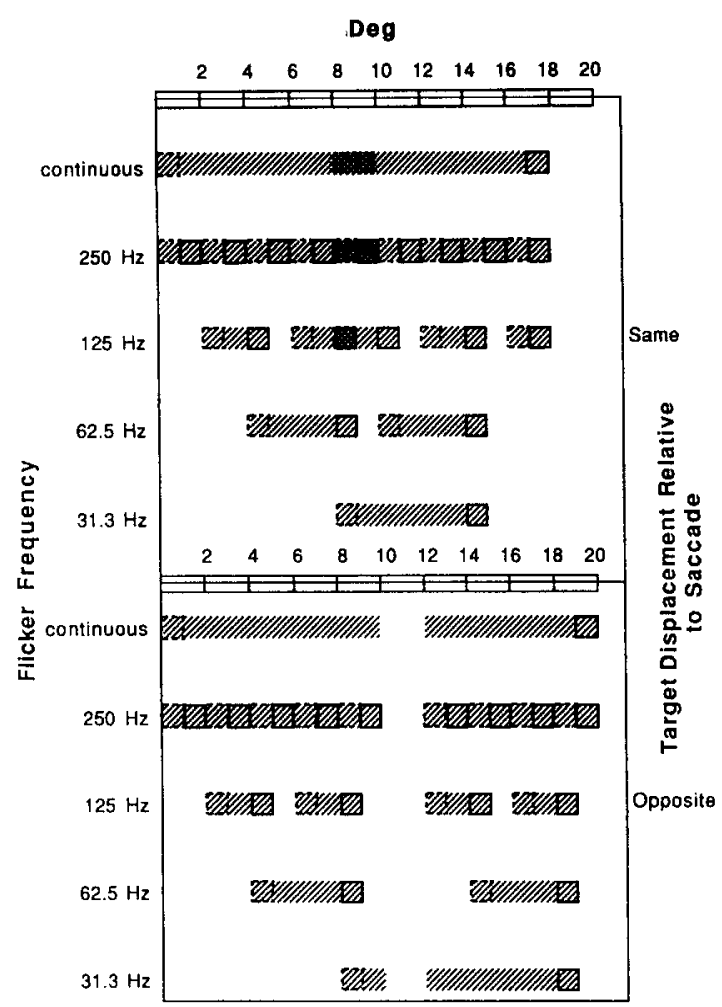

Fig. 6. Schematic retinal images of a continuous or flickered square that is displaced $2^{\circ}$ at the 10 th degree of a simulated saccade. Retinal smear is depicted for both directions of target movement and each of 4 flicker frequencies as well as continuous presentation. Display conventions as in Fig. 5. Heavy hatching in the top panel represents areas where the target overwrites the retina twice. In the $250 \mathrm{~Hz}$ condition in the "same" direction (top panel), one of the flicker samples is overwritten twice at the same retinal location. If the continuous target were moved rapidly on the screen rather than extinguished and immediately restored at the new position, the streak on the retina would remain continuous.

is displaced in the direction opposite the saccade. Because only a delayed sample is available between samples of the flickering target, retinal position of the target will lag behind the position that would have been signalled without flicker. A displacement in the same direction as the saccade, then, reduces the spatial lag, making the spatial aspect of the target more like that during continuous illumination. A displacement in the opposite direction, though, might be expected to add to the flicker-induced spatial sampling distortion. This is consistent with what we found; displacement was more detectable with slower flicker, and displacements in the direction opposite the saccade were more easily detected. Earlier studies (Bridgeman et al., 1975; Stark et al., 1976) implied that the mismatch between retinal and extraretinal signals had to exceed a threshold before intrasaccadic dis- placements could be perceived. If flicker adds a bias to the apparent size of the retinal signal, the current results could be interpreted in the same way as the earlier results.

Although spatial masking may be influencing our results, the conclusion drawn earlier (Bridgeman, 1983) showed that even when masking has its greatest effect, under continuous lighting during fixation, the effect is small compared to that of extraretinal signals mediating saccadic suppression of displacement. Because our saccadic suppression of displacement effects are so large $\left(1.33^{\circ}\right.$ displacements were suppressed) this suggests that masking plays only a small role in suppressing displacements. Future experiments will attempt to segregate masking from internally originating saccadic suppression components completely.

Finally, the finding that there is significant distortion of normal saccadic suppression as well as reduction of displacement thresholds even at $66 \mathrm{~Hz}$, close to the $60-72 \mathrm{~Hz}$ flicker frequencies commonly used in video display terminals (VDTs), implies that one of the causes of visual complaints associated with prolonged VDT use may be a stress on the space constancy system. Under flicker, the system behaves as though the target had undergone a subthreshold displacement in the direction opposite the saccade with each change in fixation; this subthreshold displacement was summed with the added displacement in our experiments to make detection easier when the eye moves in the direction opposite the displacement. Apparent displacement in the direction opposite the eye movement indicates a breakdown in space constancy, however; when space constancy fails (such as during fatigue, etc.), the world seems to move in a direction opposite the eye. Even when this displacement fails to reach perceptual threshold, it may affect spatial processing.

In this way flicker results in what can be interpreted as a partial breakdown in space constancy, and such complaints as "eyestrain" may result from the continual need to achieve constancy despite this disturbance. "Eye fatigue" is the most frequently reported visual complaint for both transient and persistent complaint groups in surveys of VDT users (Rossignol, Morse, Summers, Pagnotto, 1987). It may be possible to design VDT display formats that do not result in distortions of space constancy, and that will be easier and less fatiguing to use. 
Acknowledgements-Supported by AFOSR grant 90-0095, a UCSC Council of Provosts research grant, the Wm T. Massaro research award, and by a University of California Seed Money Grant to Bruce Bridgeman. We thank Mark Goettsche for technical support.

\section{REFERENCES}

Bridgeman, B. (1983). Mechanisms of space constancy. In Hein, A. \& Jeannerod, M. (Eds), Spatially oriented behaviour (pp. 623-632). New York: Springer.

Bridgeman, B. \& Stark, L. (1979). Omnidirectional increase in threshold for image shifts during saccadic eye movements. Perception and Psychophysics, 25, 241-243.

Bridgeman, B. \& Stark, L. (1991). Ocular proprioception and efference copy in registering visual direction. Vision Research, 31, 1903-1913.

Bridgeman, B., Hendry, D. \& Stark, L. (1975). Failure to detect displacement of the visual world during saccadic eye movements. Vision Research, 15, 719-722.

Brooks, \& Fuchs, A. (1975). Influence of stimulus parameters on visual sensitivity during saccadic eye movement. Vision Research, 15, 1389-1398.

Dodge, R. (1900). Visual perception during eye movement. Psychological Review, 7, 454-465.

Festinger, L. \& Holtzman, J. D. (1978). Retinal image smear as a source of information about magnitude of eye movement. Journal of Experimental Psychology: Human perception and Performance, 4, 473-585.

Gauthier, G., Nommay, D. \& Vercher, J.-L. (1990). Ocular muscle proprioception and visual localization of targets in man. Brain, 113, 1857-1871.

Grüsser, O.-J. (1972). Metacontrast and the perception of the visual world. European Journal of Physiology, 332 (Suppl.), R98.

Grüsser, O.-J., Krizic, A. \& Weiss, L.-R. (1987). Afterimage movement during saccades in the dark. Vision Research, $27,215-226$.

von Holst, E. \& Mittelstaedt, H. (1950). Das Reafferenzprinzip. Wechselwirkungen zwischen Zentralnervensystem und Peripherie. Naturwissenschaften, 37, 464-476.

Keller, P. (1983). Recent phosphor screen registrations and the worldwide phosphor type designation system. Proceedings of the Society for Information Display, 24, 323-328.
Latour, P. (1962). Visual threshold during eye movements. Vision Research, 2, 261-262.

Li, W. \& Matin, L. (1990). Saccadic suppression of displaccment: Influence of postsaccadic exposure duration and of saccadic stimulus elimination. Vision Research, 30 , 945-955.

Mack, A. (1970). An investigation of the relationship between eye and retinal image movement in the perception of movement. Perception and Psychophysics, 8, 291-298.

Matin, L. (1972). Eye movements and perceived visual direction. In Jameson, D. \& Hurvich, L. (Eds), Handbook of sensory physiology (Vol. 7/4). Heidelberg: Springer.

Matin, E., Clymer, A. \& Matin, L. (1972). Metacontrast and saccadic suppression. Science, 178, 179-182.

Pola, J. \& Wyatt, H. J. (1989). The perception of target motion during smooth pursuit eye movements in the open-loop condition: Characteristics of retinal and extraretinal signals. Vision Research, 29, 471-483.

Rossignol, A. M., Morse, E. P., Summers, V. M. \& Pagnotto, L. (1987). Video display terminal use and reported health symptoms among Massachusetts clerical workers. Journal of Occupational Medicine, 29. $112-118$.

Sperry, R. W. (1950). Neural basis of the spontaneous optokinetic response produced by visual inversion. Jour nal of Comparative Psychology and Physiology, 43. $482-489$.

Stark, L., Kong, R., Schwartz, S., Hendry, D. \& Bridgeman, B. (1976). Saccadic suppression of image displacement. Vision Research, 16, 1185-1187.

Teuber, H.-L. (1960). Perception. In Field, J. \& Magoun, H. W. (Eds), Handbook of physiology Sect. I: Neurophysiology, Vol. III. Washington, D.C.: American Physiological Society, pp. 1595-1668.

Volkmann, F., Riggs, L., White, K. D. \& Moore, R. K (1978). Contrast sensitivity during saccadic eye move ments. Vision Research, 18, 1193-1199.

Yasui, S. \& Young, L. R. (1975a). Eye movements during after-image tracking under sinusoidal and random vestibular stimulation. In Lennerstrand, G. \& Bach-yRita, P. (Eds), Basic mechanisms of ocular motility and their clinical implications. New York: Pergamon Press.

Yasui, S. \& Young, L. R. (1975b). Perceived visual motion as effective stimulus to pursuit eye movement system. Science, 190, 906-908. 